\title{
An efficient and green procedure for the synthesis of highly substituted polyhydronaphthalene derivatives via a one-pot, multi-component reaction in aqueous media
}

\author{
Adeleh Moshtaghi Zonouz ${ }^{a^{*}}$, Issa Eskandari ${ }^{a}$ and Behrouz Notash ${ }^{b}$
}

${ }^{a}$ Chemistry Department, Faculty of Science, Azarbaijan Shahid Madani University, Tabriz, Iran

${ }^{b}$ Department of Chemistry, Shahid Beheshti University, G.C. Tehran 1983963113, Iran

\begin{tabular}{lll}
\hline C H R O N I C L E & A B S T R A C T \\
\cline { 1 - 3 } \cline { 3 - 4 } $\begin{array}{l}\text { Article history: } \\
\text { Received January 21, 2015 }\end{array}$ & & $\begin{array}{l}\text { A simple, efficient and green one-pot, four-component synthesis of highly substituted } \\
\text { Received in revised form }\end{array}$ \\
$\begin{array}{l}\text { March 29, 2015 } \\
\text { Accepted 29 April 2015 }\end{array}$ & $\begin{array}{l}\text { short reaction times, high yields, mild reaction conditions, operational simplicity and } \\
\text { environmentally benign. }\end{array}$
\end{tabular}

Accepted 29 April 2015

Available online

29 April 2015

Keywords:

Polyhydronaphthalene

2,6-dicyanoanilines

Multi-component reaction

Aqueous media

\section{Introduction}

Green Chemistry is the commonly accepted term to describe the movement towards more environmentally acceptable chemical processes and products. ${ }^{1-3}$ Given the desirable economic, safety and environmental properties of water, it is the ideal green solvent for organic reactions. On the other hand, there are many potential advantages in using water as a solvent for organic reactions, such as synthetic efficiency, simple operation, and potential for new synthetic methodologies. ${ }^{1}$

Multi-component reactions (MCRs) are convergent reactions, in which three or more starting materials react to form a product, where all or most of the atoms contribute to the newly formed product. The major advantages of MCRs include lower costs, shorter reaction times, high atom-economy, energy saving, and the avoidance of time consuming and expensive purification processes. It is established * Corresponding author.

E-mail address: adelehmz@yahoo.com (A. Moshtaghi Zonouz) 
that MCRs are often more environmentally friendly, and offer rapid access to large compound libraries with diverse functionalities. ${ }^{4-7}$

2,6-Dicyanoanilines are typical acceptor-donor-acceptor (A-D-A) systems comprising one electron-donor ( $\mathrm{NH}_{2}$ group) and two electron-acceptors (cyano groups). ${ }^{8}$ These molecular systems have attracted much attention because of their optical properties. ${ }^{9}$ They are the basis for artificial photosynthesis, ${ }^{10}$ materials presenting semi-conducting or nonlinear optical properties ${ }^{11}$ and molecular electronic devices. ${ }^{12}$ 2-Amino-1,3,3-tricyano-substituted polyhydronaphthalenes containing 2,6dicyanoamine moiety with one electron-donor and three electron-acceptors belong to typical acceptordonor-acceptor-acceptor (A-D-A-A) systems. ${ }^{13}$

2,6-Dicyanoanilines have been prepared from arylidenemalonodinitriles and 1arylethylidenemalonodinitriles in the presence of piperidine, ${ }^{14}$ ethanediamine, ${ }^{15}$ triethylamine, ${ }^{16}$ $\mathrm{NaOH},{ }^{17}$ or under microwave irradiation. ${ }^{18}$ The reaction between propanedinitrile and $\alpha, \beta$-unsaturated ketones could also give 2,6-dicyanoanilines. The reported yields of 2,6-dicyanoanilines were poor, ${ }^{19}$ and all the above-mentioned reactions were performed in organic solvents. Also synthesis of the onedonor poly-acceptors systems containing 2,6-dicyanoamine moiety have been reported in aqueous media in the presence of catalysts such as TEBAC (triethylbenzylammonium chloride), ${ }^{20} \mathrm{ZnTiO}_{3}$ nanopowder, ${ }^{13}$ and chitosan. ${ }^{21}$ The synthesis of the A-D-A-A type of compounds have also been reported via three-component reactions in ionic liquids. ${ }^{22}$

\section{Results and Discussion}

As part of our efforts to develop new synthetic methods, ${ }^{73-25}$ herein we wish to demonstrate a simple, versatile, and environmentally friendly one-pot three-component reaction for the combinatorial library synthesis of 2-amino-1,3-dicyano-substituted polyhydronaphthalenes in aqueous media in the presence of ammonium acetate as a safe, inexpensive and nontoxic catalyst (Scheme 1 and Table 1).
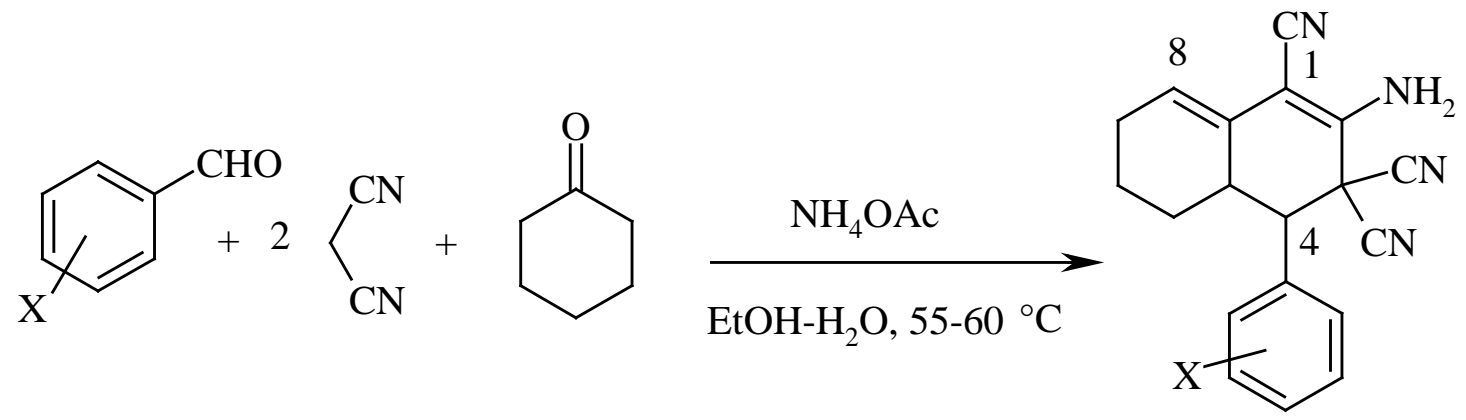

3a-i

Scheme 1. One-pot, four-component synthesis of A-D-A-A systems in aqueous media

Initially, we carried out the MCR of malononitrile, aromatic aldehydes and cyclohexanone in the presence of ammonium acetate in water:ethanol $(1: 1)$ at $55-60{ }^{\circ} \mathrm{C}$. After completion of the reaction (TLC monitoring), TLC indicated the mixture of products, so the reaction was conducted in two stages, first of all treatment of arylaldehyde and malononitrile (1:1 molar ratio) in water:ethanol for 30 min at $55-60{ }^{\circ} \mathrm{C}$ gave the benzylidene malononitrile 1 which, without isolation, was directly treated with ammonium acetate, another mole of malononitrile and cyclohexanone. Stirring was maintained for an additional 2 hours. The precipitated solid was filtered and purified by recrystallization from EtOH to give the products $\mathbf{3 a - i}$ in good to excellent yields (Table 1). In the absence of ammonium acetate, the reaction did not yield the expected product. 
Table 1. One-pot, four-component synthesis of polyhydronaphthalenes in aqueous media ${ }^{\mathrm{a}}$

\begin{tabular}{lllll}
\hline Entry & $\mathbf{X}$ & Product & Yield $(\%)$ & Mp $\left({ }^{\circ} \mathrm{C}\right){ }^{\mathrm{b}}$ \\
\hline 1 & $\mathrm{H}$ & $\mathbf{3 a}$ & 90 & $250-251\left(\right.$ Lit. $^{16}$ 250) \\
2 & $2-\mathrm{Me}$ & $\mathbf{3 b}$ & 93 & $250-251$ \\
3 & $2-\mathrm{b} e$ & $\mathbf{3 c}$ & 87 & $248-249$ \\
4 & $3-\mathrm{Me}$ & $\mathbf{3 d}$ & 87 & $194-195$ \\
5 & $2-\mathrm{Br}$ & $\mathbf{3 e}$ & 88 & $235-236$ \\
6 & $4-\mathrm{Br}$ & $\mathbf{3 f}$ & 92 & $236-238$ (Lit. $^{22}$ 264-265) \\
7 & $2-\mathrm{Cl}$ & $\mathbf{3 g}$ & 86 & $244-245$ (Lit. $^{22}$ 279-280) \\
8 & $3-\mathrm{Cl}$ & $\mathbf{3 h}$ & 89 & $239-240$ (Lit. $^{22}$ 122-123) \\
9 & $3-\mathrm{OH}$ & $\mathbf{3 i}$ & 91 & $212-213$ \\
\hline
\end{tabular}

${ }^{a}$ All reactions were carried out in ethanol-water using aldehyde (1 mmol), cyclohexanone (1 mmol), malononitrile (2 mmol), ammonium acetate $(1.3 \mathrm{mmol}) .{ }^{\mathrm{b}}$ Isolated yields after recrystallization.

The formation of polyhydronaphthalene derivatives 3a-i can be rationalized by the following tandem reactions: 1) formation of benzylidine malononitrile $\mathbf{1}$ by Knoevenagal condensation of aromatic aldehyde and malononitrile; 2) formation of $\mathbf{2}$ by Knoevenagal condensation of cyclohexanone and malononitrile; and 3) Michael addition of $\mathbf{2}$ to $\mathbf{1}$, followed by cyclization and tautomerization (Scheme 2). Ammonium acetate acts as a catalyst in the Knovenagel condensation and Michael addition reactions.

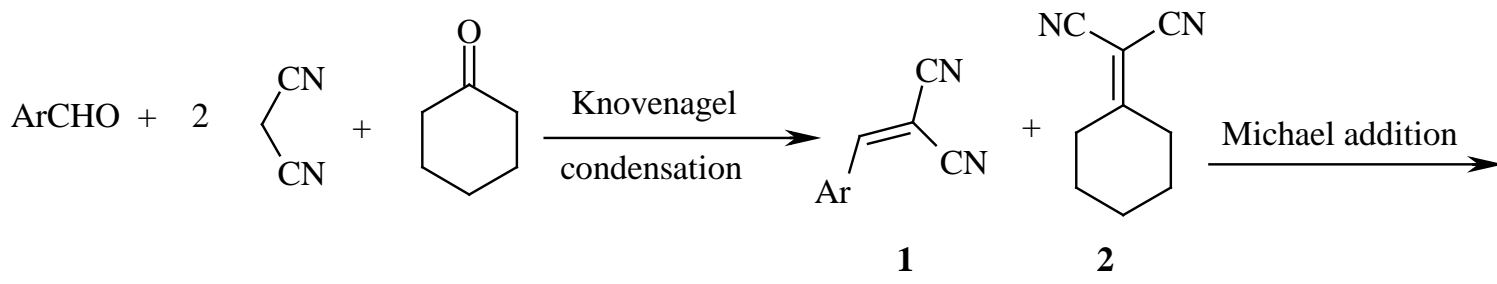<smiles>N#CC(C#N)=C1CCCCC1C(Br)C#N</smiles>

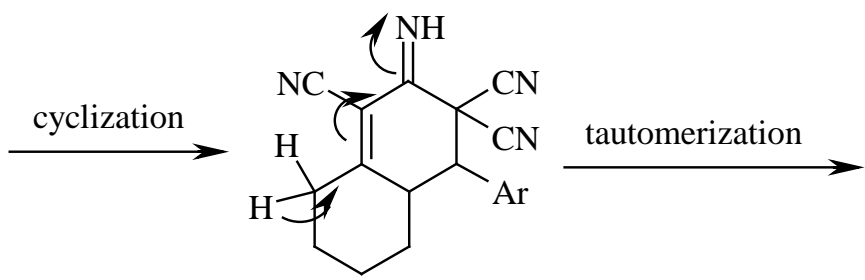<smiles>N#CC1=C(N)C(C#N)(C#N)C(Br)C2CCCC=C12</smiles>

Scheme 2. Proposed mechanism for the formation of products 3<smiles>[X]c1cccc(C2C3=C(CCCC3)C(C#N)=C(N)C2(C#N)C#N)c1</smiles>

A<smiles>[X]c1cccc(C2C3CCCC=C3C(C#N)=C(N)C2(C#N)C#N)c1</smiles>

B

Fig. 1. Two different structures for 2-amino-1,3-dicyano-substituted polyhydronaphthalenes 
Notably, two slightly different structures $\mathbf{A}^{13,20,26}$ and $\mathbf{B}^{16,21,22}$ have been reported for the compounds 3. Thus, we eventually solve the issue of those conflicting reports. By means of elemental and spectral as well as X-ray crystallographic analysis. We determined that structure of 2-amino-4-(2chlorophenyl)-4a,5,6,7-tetrahydro-4H-naphthalene-1,3,3-tricarbonitrile (3g) in fact corresponds to formulae B. ${ }^{27,28}$ Figure 2 shows the structure and the atomic numbering scheme used for compound $3 g$.

The X-ray results indicate that the bond lengths of C12-C14 (1.476 $\AA$ ) and C14-C19 (1.517 $\AA$ ) approximate a C-C single bond, whereas C11-C12 (1.347 $\AA$ ) and C14-C15 (1.326 $\AA$ ) correspond to C$\mathrm{C}$ double bond. Hence, with no further doubts performed crystallographic analysis confirmed the structure $\mathbf{B}$.

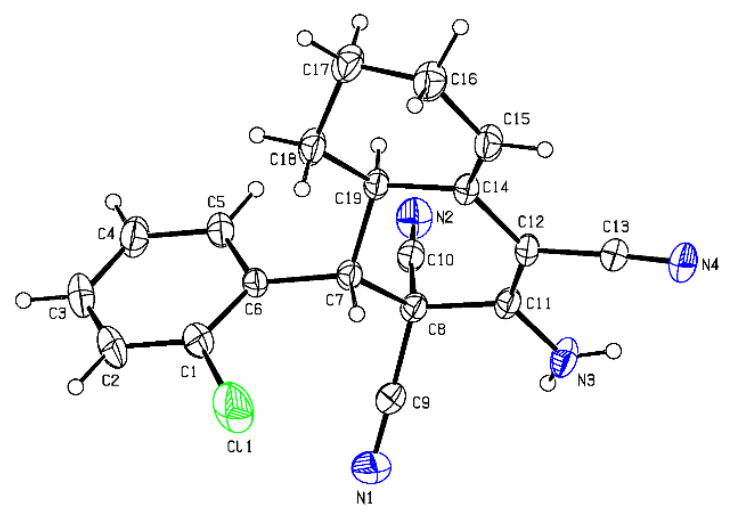

Fig. 2. ORTEP diagram of 3g

\section{Conclusions}

In summary, we have developed an efficient and simple one-pot method for the synthesis of polyhydronaphthalenes via multicomponent reaction in 50\% aqueous $\mathrm{EtOH}$ in the presence of ammonium acetate. The features of this procedure include mild reaction condition, high yields, short reaction time, simple purification and environmentally benign.

\section{Acknowledgements}

The authors sincerely acknowledge the Research Office of Azarbaijan Shahid Madani University for financial support.

\section{Experimental}

\subsection{General}

The ${ }^{1} \mathrm{H}$ - and ${ }^{13} \mathrm{C}-\mathrm{NMR}$ spectra were taken on a Bruker SP-250 AVANCE spectrometers. The IR spectra were recorded on a Bruker PS-15 spectrometer. The elemental analyses were performed on a Carlo-Erba $1104 \mathrm{CHN}$ analyzer. The melting points were measured on an Electrothermal 9100 apparatus in open capillaries without correction. All the commercial reagents were used without prior purification.

\subsection{Typical procedure for the synthesis of Polyhydronaphthalenes 3a-i.}

A mixture of benzaldehyde ( $1 \mathrm{mmol}, 0.1 \mathrm{ml})$, malononitrile $(1 \mathrm{mmol}, 66 \mathrm{mg})$ in $50 \%$ aqueous ethanol (3 ml) was stirred at $55-60{ }^{\circ} \mathrm{C}$ for $30 \mathrm{~min}$. Next, ammonium acetate $(1.3 \mathrm{mmol}, 100 \mathrm{mg})$, 
malononitrile $(1 \mathrm{mmol}, 66 \mathrm{mg})$ and cyclohexanone $(1 \mathrm{mmol}, 0.1 \mathrm{~mL})$ were added. The mixture was stirred until completion of the reaction as indicated by TLC (2 hours). After cooling, the precipitated white solid was filtered, washed with water and recrystallized from EtOH. Compound 2a was obtained as pale yellow crystals.

4.2.1. 2-Amino-4a,5,6,7-tetrahydro-4-phenyl-4H-naphthalene-1,3,3-tricarbonitrile (3a). mp 250-251 ${ }^{\circ} \mathrm{C}$ (recrystallized from EtOH); yield: 0.270 g (90\%). IR (KBr) 3447, 3359, 3001, 2963, 2920, 2858, 2844, 2217, 1632, 1617, 1599, 1492, 1390, 1271, 1028, $754 \mathrm{~cm}^{-1} ;{ }^{1} \mathrm{H}$ NMR (250 MHz, DMSO-d $): \delta$ 0.65-0.85 (m, 1H, CH), 1.36-1.43 (m, 2H, CH 2$), 1.62(\mathrm{~m}, 1 \mathrm{H}, \mathrm{CH}), 1.90-2.20\left(\mathrm{~m}, 2 \mathrm{H}, \mathrm{CH}_{2}\right), 2.87(\mathrm{~m}$, $1 \mathrm{H}, \mathrm{CH}), 3.50$ (d, $J=10 \mathrm{~Hz}, 1 \mathrm{H}, \mathrm{CH}), 5.70(\mathrm{~s}, 1 \mathrm{H}, \mathrm{CH}), 7.24-7.70\left(\mathrm{~m}, 7 \mathrm{H}, \mathrm{NH}_{2}\right.$ and $\left.\mathrm{CH}_{\text {arom}}\right) \mathrm{ppm} ;{ }^{13} \mathrm{C}$ NMR (62.5 MHz, DMSO-d $): \delta 21.39\left(\mathrm{CH}_{2}\right), 25.23\left(\mathrm{CH}_{2}\right), 27.43\left(\mathrm{CH}_{2}\right), 34.28(\mathrm{CH}), 43.28(\mathrm{CH})$, 50.92 (C), $81.90(\mathrm{C}), 112.97(\mathrm{CN}), 116.61(\mathrm{CN}), 120.37(\mathrm{CH}), 127.37(\mathrm{CH}), 129.00(\mathrm{CH}), 132.76(\mathrm{C})$, 135.02 (C), 143.96 (C) ppm; Anal. Calcd. for $\mathrm{C}_{19} \mathrm{H}_{16} \mathrm{~N}_{4}$ : C, 75.98; H, 5.37; N, 18.61. Found: C, 75.89; H, 5.48; N, 18.99 .

4.2.2. 2-Amino-4a,5,6,7-tetrahydro-4-(2-methylphenyl)naphthalene-1,3,3(4H)-tricarbonitrile (3b). Yellow crystals; mp 250-251 ${ }^{\circ} \mathrm{C}$ (recrystallized from EtOH); yield: 0.292 g (93 \%). IR (KBr) 3446, 3357, 3001, 2950, 2920, 2858, 2216, 1633, 1618, 1599, 1446, 1391, 1272, $732 \mathrm{~cm}^{-1}$; ${ }^{1} \mathrm{H}$ NMR $(250$ MHz, DMSO-d 6 ): $\delta$ 0.79-0.88 (m, 1H, CH), 1.20-1.47 (m, 2H, CH2), 1.59 (m, 1H, CH), 1.90-2.20 (m, $\left.2 \mathrm{H}, \mathrm{CH}_{2}\right), 2.35$ (s, 3H, CH $), 2.76(\mathrm{~m}, 1 \mathrm{H}, \mathrm{CH}), 3.64(\mathrm{~d}, J=12.5 \mathrm{~Hz}, 1 \mathrm{H}, \mathrm{CH}), 5.71(\mathrm{~s}, 1 \mathrm{H}, \mathrm{CH}), 7.28-$ $7.57\left(\mathrm{~m}, 6 \mathrm{H}, \mathrm{NH}_{2}\right.$ and $\mathrm{CH}$ arom) ppm; ${ }^{13} \mathrm{C} \mathrm{NMR}\left(62.5 \mathrm{MHz}, \mathrm{DMSO}-\mathrm{d}_{6}\right): \delta 20.16\left(\mathrm{CH}_{3}\right), 21.46\left(\mathrm{CH}_{2}\right)$, $25.28\left(\mathrm{CH}_{2}\right), 27.07\left(\mathrm{CH}_{2}\right), 35.42(\mathrm{CH}), 42.59(\mathrm{CH}), 45.94(\mathrm{C}), 82.00(\mathrm{C}), 112.72(\mathrm{CN}), 113.15(\mathrm{CN})$, $116.68(\mathrm{CN}), 120.66(\mathrm{CH}), 126.83(\mathrm{CH}), 127.56(\mathrm{CH}), 128.87(\mathrm{C}), 129.42(\mathrm{CH}), 131.53(\mathrm{CH}) 133.49$ (C), 138.69 (C), 144.32 (C) ppm; Anal. Calcd. for $\mathrm{C}_{20} \mathrm{H}_{18} \mathrm{~N}_{4}$ : C, 76.41; H, 5.77; N, 17.82. Found: C, 76.80; H, 5.95; N, 17.96 .

4.2.3. 2-Amino-4a,5,6,7-tetrahydro-4-(3-methylphenyl)naphthalene-1,3,3(4H)-tricarbonitrile (3c). Yellow crystals; mp 194-195 ${ }^{\circ} \mathrm{C}$ (recrystallized from EtOH); yield: 0.273 g (87 \%). IR (KBr) 3456, 3395, 3166, 2950, 2935, 2858, 2208, 1668, 1641, 1597, 1394, 1267, 1046, 873, 799, $743 \mathrm{~cm}^{-1} ;{ }^{1} \mathrm{H}$ NMR (250 MHz, DMSO-d $): \delta 1.09$ (m, 1H, CH), 1.60-1.77 (m, 2H, CH2), $1.86(\mathrm{~m}, 1 \mathrm{H}, \mathrm{CH}), 2.20-$ 2.35 (m, 2H, CH 2$), 2.55$ (s, 3H, $\left.\mathrm{CH}_{3}\right), 2.96$ (m, 1H, CH), 3.55 (d, J = 12.5 Hz, 1H, CH), 5.94 (s, 1H, $\mathrm{CH})$, 7.28-7.57 (m, 6H, NH 2 and $\mathrm{CH}$ arom) ppm; ${ }^{13} \mathrm{C}$ NMR (62.5 MHz, DMSO-d $)$ : $\delta 21.46\left(\mathrm{CH}_{2}\right)$, $24.26\left(\mathrm{CH}_{3}\right), 25.28\left(\mathrm{CH}_{2}\right), 27.17\left(\mathrm{CH}_{2}\right), 35.42(\mathrm{CH}), 42.59(\mathrm{CH}), 45.94(\mathrm{C}), 82.60(\mathrm{C}), 113.15(\mathrm{CN})$, $116.68(\mathrm{CN}), 120.66(\mathrm{CH}), 126.13(\mathrm{CH}), 127.76(\mathrm{CH}), 128.50(\mathrm{C}), 130.12(\mathrm{CH}), 133.53(\mathrm{CH}) 135.42$ (C), 139.79 (C), 145.43(C); Anal. Calcd. for $\mathrm{C}_{20} \mathrm{H}_{18} \mathrm{~N}_{4}$ : C, 76.41; H, 5.77; N, 17.82. Found: C, 76.70; H, 5.95; N, 17.86 .

4.2.4. 2-Amino-4a,5,6,7-tetrahydro-4-(2-methoxyphenyl)naphthalene-1,3,3(4H)-tricarbonitrile (3d). Yellow crystals; mp 248-249 ${ }^{\circ} \mathrm{C}$ (recrystallized from EtOH); yield: $0.287 \mathrm{~g}$ (87 \%). IR (KBr) 3446, 3358, 2944, 2217, 1633, 1618, 1599, 1493, 1464, 1391, 1252, 1122, 810, $754 \mathrm{~cm}^{-1}$; ${ }^{1} \mathrm{H}$ NMR $(250$ MHz, DMSO-d6): $\delta$ 0.66-0.75 (m, 1H, CH), 1.30-1.47 (m, 2H, CH2), 1.59 (m, 1H, CH), 2.00-2.20 (m, 2H, $\left.\mathrm{CH}_{2}\right), 2.75$ (m, 1H, CH), 3.79 (s, 3H, OCH $), 3.84$ (d, J = $\left.12.5 \mathrm{~Hz}, 1 \mathrm{H}, \mathrm{CH}\right), 5.70(\mathrm{~s}, 1 \mathrm{H}, \mathrm{CH})$, 6.92-7.51 (m, 6H, NH 2 and $\mathrm{CH}$ arom) ppm; ${ }^{13} \mathrm{C}$ NMR (62.5 MHz, DMSO-d $): \delta 21.36\left(\mathrm{CH}_{2}\right), 25.26$ $\left(\mathrm{CH}_{2}\right), 27.22\left(\mathrm{CH}_{2}\right), 34.36(\mathrm{CH}), 42.48(\mathrm{CH}), 42.70(\mathrm{CH}), 56.22\left(\mathrm{OCH}_{3}\right), 81.95(\mathrm{C}), 112.19(\mathrm{CN})$, $112.47(\mathrm{CN}), 113.08(\mathrm{CN}), 116.56(\mathrm{CH}), 121.03(\mathrm{CH}), 122.61(\mathrm{CH}), 128.50(\mathrm{C}), 129.34(\mathrm{CH}), 130.53$ (CH) 144.22 (C), 158.22 (C) ppm; Anal. Calcd. for $\mathrm{C}_{20} \mathrm{H}_{18} \mathrm{~N}_{4} \mathrm{O}$ : C, 72.71; H, 5.49; N, 16.96. Found: C, 72.38; H, 5.74; N, 16.99 .

4.2.5. 2-Amino-4a,5,6,7-tetrahydro-4-(2-bromophenyl)naphthalene-1,3,3(4H)-tricarbonitrile (3e). Yellow crystals; mp 235-236 ${ }^{\circ} \mathrm{C}$ (recrystallized from EtOH); yield: $0.333 \mathrm{~g}$ (88 \%). IR (KBr) 3426, 3385, 3166, 2950, 2933, 2214, 1641, 1557, 1456, 1250, 1026, $751 \mathrm{~cm}^{-1}$; ${ }^{1} \mathrm{H}$ NMR (250 MHz, DMSOd6): $\delta 1.02(\mathrm{~m}, 1 \mathrm{H}, \mathrm{CH}), 1.50-1.77\left(\mathrm{~m}, 2 \mathrm{H}, \mathrm{CH}_{2}\right), 1.86(\mathrm{~m}, 1 \mathrm{H}, \mathrm{CH}), 2.25-2.35(\mathrm{~m}, 2 \mathrm{H}, \mathrm{CH} 2), 3.20(\mathrm{~m}$, 
1H, CH), 4.10 (d, $J=12.5 \mathrm{~Hz}, 1 \mathrm{H}, \mathrm{CH}), 5.99$ (s, 1H, CH), 7.51-7.81 (m, 6H, $\mathrm{NH}_{2}$ and $\mathrm{CH}$ arom) ppm; Anal. Calcd. for $\mathrm{C}_{19} \mathrm{H}_{15} \mathrm{BrN}_{4}$ : C, 60.17; H, 3.99; N, 14.77. Found: C, 60.50; H, 3.95; N, 14.96.

4.2.6. 2-Amino-4a,5,6,7-tetrahydro-4-(4-bromophenyl)naphthalene-1,3,3(4H)-tricarbonitrile (3f). Yellow crystals; mp 236-238 ${ }^{\circ} \mathrm{C}$ (recrystallized from EtOH); yield: 0.348 g (92 \%). IR (KBr) 3422, 3345, 3156, 2926, 2211, 1644, 1601, 1489, 1391, 1278, 1076, 1011, 834, 805, $751 \mathrm{~cm}^{-1}$; ${ }^{1} \mathrm{H}$ NMR $(250$ MHz, DMSO-d6): $\delta 1.05$ (m, 1H, CH), 1.60-1.79 (m, 2H, CH2), $1.93(\mathrm{~m}, 1 \mathrm{H}, \mathrm{CH}), 2.29-2.44(\mathrm{~m}, 2 \mathrm{H}$, $\mathrm{CH}_{2}$ ), 3.02 (m, 1H, CH), 3.85 (d, $\left.J=12.5 \mathrm{~Hz}, 1 \mathrm{H}, \mathrm{CH}\right), 5.96$ (s, 1H, CH), 7.61-7.95 (m, 6H, NH 2 and $\mathrm{CH}$ arom) ppm; Anal. Calcd. for $\mathrm{C}_{19} \mathrm{H}_{15} \mathrm{BrN}_{4}$ : C, 60.17; H, 3.99; N, 14.77. Found: C, 60.44; H, 4.15; N, 15.03 .

4.2.7. 2-Amino-4a,5,6,7-tetrahydro-4-(2-chlorophenyl)naphthalene-1,3,3(4H)-tricarbonitrile (3g). Yellow crystals; mp 244-245 ${ }^{\circ} \mathrm{C}$ (recrystallized from EtOH); yield: $0.288 \mathrm{~g}$ (86 \%). IR (KBr) 3446, 3357, 3166, 2948, 2214, 1633, 1595, 1557, 1478, 1391, 1250, 1056, 776, $702 \mathrm{~cm}^{-1}$; ${ }^{1} \mathrm{H}$ NMR $(250$ MHz, DMSO-d6): $\delta=1.05$ (m, 1H, CH), 1.57-1.67 (m, 2H, CH2), $1.86(\mathrm{~m}, 1 \mathrm{H}, \mathrm{CH}), 2.26-2.36(\mathrm{~m}, 2 \mathrm{H}$, $\mathrm{CH}_{2}$ ), 3.06 (m, 1H, CH), 4.10 (d, $\left.J=12.5 \mathrm{~Hz}, 1 \mathrm{H}, \mathrm{CH}\right), 5.99$ (s, 1H, CH), 7.52-7.86 (m, 6H, NH 2 and $\mathrm{CH}$ arom) ppm; Anal. Calcd. for $\mathrm{C}_{19} \mathrm{H}_{15} \mathrm{ClN}_{4}$ : C, 68.16; H, 4.52; N, 16.73. Found: C, 68.40; H, 4.95; N, 17.03 .

4.2.8. 2-Amino-4a,5,6,7-tetrahydro-4-(3-chlorophenyl)naphthalene-1,3,3(4H)-tricarbonitrile (3h). Yellow crystals; mp 239-240 ${ }^{\circ} \mathrm{C}$ (recrystallized from EtOH); yield: 0.298 g (89 \%). IR (KBr) 3420, 3342, 3235, 2925, 2210, 1649, 1603, 1573, 1478, 1393, 1279, 1092, 847, 797, 748, $705 \mathrm{~cm}^{-1} ;{ }^{1} \mathrm{H}$ NMR (250 MHz, DMSO-d6): $\delta=1.05$ (m, 1H, CH), 1.64 (m, 2H, CH2), 1.86 (m, 1H, CH), 2.34-2.41 (m, 2H, $\left.\mathrm{CH}_{2}\right), 2.99(\mathrm{~m}, 1 \mathrm{H}, \mathrm{CH}), 3.75(\mathrm{~d}, J=12.5 \mathrm{~Hz}, 1 \mathrm{H}, \mathrm{CH}), 5.95(\mathrm{~s}, 1 \mathrm{H}, \mathrm{CH}), 7.52-7.86\left(\mathrm{~m}, 6 \mathrm{H}, \mathrm{NH}_{2}\right.$

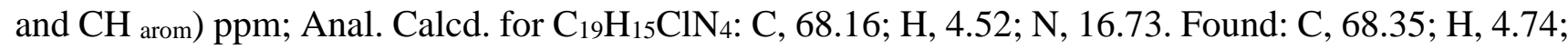
N, 16.96.

4.2.9. 2-Amino-4a,5,6,7-tetrahydro-4-(3-hydroxyphenyl)naphthalene-1,3,3(4H)-tricarbonitrile (3i). White crystals, mp 212-213 ${ }^{\circ} \mathrm{C}$ (recrystallized from EtOH); yield: $0.288 \mathrm{~g}$ (91 \%). IR (KBr) 3449, 3412, 3235, 2929, 2192, 1675, 1649, 1533, 1350, 811, 737, $690 \mathrm{~cm}^{-1} ;{ }^{1} \mathrm{H}$ NMR (250 MHz, DMSO-d $): \delta=$ 1.05-1.12 (m, 1H, CH), 1.54-1.70 (m, 2H, CH2), $1.89(\mathrm{~m}, 1 \mathrm{H}, \mathrm{CH}), 2.34-2.44$ (m, 2H, $\left.\mathrm{CH}_{2}\right), 2.90(\mathrm{~m}$, $1 \mathrm{H}, \mathrm{CH}), 3.51(\mathrm{~d}, J=12.5 \mathrm{~Hz}, 1 \mathrm{H}, \mathrm{CH}), 5.93(\mathrm{~s}, 1 \mathrm{H}, \mathrm{CH}), 7.02-7.81(\mathrm{~m}, 7 \mathrm{H}, \mathrm{NH} 2, \mathrm{OH}$ and $\mathrm{CH}$ arom $)$ ppm; ${ }^{13} \mathrm{C}$ NMR (62.5 MHz, DMSO-d $): \delta 21.32\left(\mathrm{CH}_{2}\right), 25.27\left(\mathrm{CH}_{2}\right), 27.20\left(\mathrm{CH}_{2}\right), 34.36(\mathrm{CH}), 41.91$ (CH), $42.70(\mathrm{C}), 81.95(\mathrm{C}), 113.21(\mathrm{CH}), 112.76(\mathrm{CN}), 113.38(\mathrm{CN}), 116.30(\mathrm{CH}), 121.20(\mathrm{CH}), 122.64$ (CH), 127.20 (C), 128.94 (CH), 130.58 (C) 144.22 (C), 158.22 (C) ppm; Anal. Calcd. for $\mathrm{C}_{19} \mathrm{H}_{16} \mathrm{~N}_{4} \mathrm{O}$ : C, 72.13; H, 5.10; N, 17.71. Found: C, 72.20; H, 5.15; N, 17.93.

\section{References}

1. (a) Ameta S. C., Ameta R. (2013) Green Chemistry: Fundamentals and Applications. CRC press; (b) Li C-J., Chan T-H. (2007) Comprehensive Organic Reactions in Aqueous Media. Eds. John Wiley \& Sons.

2. Chanda A., Fokin V. V. (2009) Organic synthesis “on water”. Chem. Rev. 109, 725-748.

3. World Commission on the Environment and Development (WCED), Our Common Future Oxford, Oxford University Press, 1987, p. 43.

4. Zhu J., Bienaymé H. (2005) Multicomponent Reactions, Wiley-VCH, Weinheim.

5. Ruijter E., Scheffelaar R., Orru R. V. A. (2011) Multicomponent Reaction Design in the quest for molecular complexity and diversity. Angew. Chem., Int. Ed. 50, 6234-6246.

6. Bannwarth W., Felder E. (2000) Combinatorial Chemistry. Wiley-VCH, Weinheim.

7. Moshtaghi Z. A., Eskandari I., Khavasi H. R. (2012) A Green and convenient approach for the synthesis of methyl 6-amino-5-cyano-4-aryl-2,4-dihydropyrano[2,3-c]pyrazole-3-carboxylates via a one-pot, multi-component reaction in water. Tetrahedron Lett. 53, 5519-5522. 
8. Balwant S. J., Keshwal S., Rajguru D., and Bhagwat V. W. (2012) A simple and clean synthesis of polysubstituted 2,6-dicyanoanilines catalyzed by KF/alumina. J. Korean Chem. Soc. 56, 712715.

9. (a) Sepiol J., Milart P. (1985) Elimination of the nitrile group from o-aminonitriles-IV: A new and efficient synthesis of 3,5-diarylaminobenzenes from arylidenemalonodinitriles and 1arylethylidenemalonodinitriles. Tetrahedron 41, 5261-5265; (b) Griffiths J., Lockwood M., Roozpeikar B. (1977) Orientation effects in the benzene chromophore bearing one donor and two acceptor groups. Electronic absorption spectra of the dicyanoanilines. J. Chem. Soc., Perkin Trans. 2, 1608-1609.

10. (a) Kurreck H., Huber M. (1995) Model reactions for photosynthesis—photoinduced charge and energy transfer between covalently linked porphyrin and quinone units. Angew. Chem., Int. Ed. 34, 849-866. (b) Long N. J. (1995) Organometallic compounds for nonlinear optics-the search for enlightenment. Angew. Chem., Int. Ed. 34, 21-38.

11. (a) Nalwa H. S. (1993) Organic materials for third-order nonlinear optics. Adv. Mater. 5, 341-358.

(b) Wong M. S., Bosshard C., Pan F., Gunter P. (1996) Non-classical donor-acceptor chromophores for second order nonlinear optics. Adv. Mater. 8, 677-680.

12. Metzger R. M., Panetta C. (1991) The quest for unimolecular rectifiers. New. J. Chem. 15, 209221.

13. Das P., Butcher R. J., Mukhopadhyay C. (2012) Zinc titanate nanopowder: an advanced nanotechnology based recyclable heterogeneous catalyst for the one-pot selective synthesis of selfaggregated low-molecular mass acceptor-donor-acceptor-acceptor systems and acceptor-donoracceptor triads. Green Chem. 14, 1376-1387.

14. Milart, P.; Wilamowski, J.; Sepiol, J. J. (1998) Synthesis of di- and triamino-1,1':3", 1"-terphenyls from arylethylidene and arylidenemalonodinitriles. Tetrahedron 54, 15643-15656.

15. Wang J., Li Q., Qi C., Liu Y., Ge Z., Li R. (2010) Primary 1,2-diamine catalysis III: an unexpected domino reaction for the synthesis of multisubstituted cyclohexa-1,3-dienamines. Org. Biomol. Chem. 8, 4240-4242.

16. El-Sakka I. A., El-Kousy S. M., Kandil Z. E. (1991) Nitriles in organic synthesis: The reaction of cinnamonitriles with cyclohexanone and acetylacetone. J. Prakt. Chem. 333, 345-350.

17. Rong L., Han H., Jiang H., Tu S. (2007) Efficient one-pot synthesis of 2-amino-4,6- diarylbenzene1,3-dicarbonitrile under solvent-free conditions. Synth. Commun. 37, 3767-3772.

18. Cui S. L., Lin X. F., Wang Y. G. (2005) Parallel synthesis of strongly fluorescent polysubstituted 2,6-dicyanoanilines via microwave-promoted multicomponent reaction. J. Org. Chem. 70, 28662869.

19. (a) Victory P. J., Borrell J. I., Vidal-Ferran A. (1993) A Simple synthesis of 2-methoxypyridine-3carbonitriles. Heterocycles 36, 769-776; (b) Victory P., Borrel J. I., Vidal-Ferran A., Seoane C., Soto J. L. (1991) The reaction of malononitrile with chalcone: a controversial chemical process. Tetrahedron Lett. 32, 5375-5378; (c) Victory P. J., Borrell J. I., Vidal-Ferran A., Montenegro E., Jimeno M. L. (1993) Synthesis of 4-amino-8-cyanoquinazolines from enones and enals. Heterocycles 36, 2273-2280.

20. Wang X. S., Zhang M. M., Jiang H., Tu S. J. (2007) An improved and clean procedure for the synthesis of one-donor poly-acceptors systems containing 2,6-dicyanoamine moiety in aqueous media catalyzed by TEBAC in the presence and absence of ${ }_{2} \mathrm{Cоз}$ Tetrahedron 63, 5265-5273.

21. Al-Matar H. M., Khalil K. D., Meier H., Kolshorn H., Elnagdi M. H. (2008) Chitosan as heterogeneous catalyst in Michael additions: The reaction of cinnamonitriles with active methyls, active methylenes and phenols. Arkivoc xvi, 288-301.

22. Wang X. S., Wu J. R., Zhou J., Tu S. J. (2009) Green method for the synyhesis of highly substituted cyclohexa-1,3-diene, polyhydroindene, polyhydronaphthalene, isochromene, isothiochromene, and isoquinoline derivatives in ionic liquids. J. Comb. Chem. 11, 1011-1022.

23. Moshtaghi Z. A., Baradaran H. S. (2008) Montmorillonite K10 Clay: An efficient catalyst for Hantzsch synthesis of 1,4-dihydropyridine derivatives. Synth. Commun. 38, 290-296. 
24. Moshtaghi Z. A., Sahranavard, N. (2010) Synthesis of 1,4-dihydropyridine derivatives under aqueous media. EJOC 7(S1), S372-S376.

25. Moshtaghi Z. A., Eskandari I., Moghani D. (2012) Acceleration of multicomponent reactions in aqueous medium: multicomponent synthesis of a 4H-pyran library. Chem. Sci. Trans. 1, 91-102.

26. Elgemeie G. E. H., Regaila H. A., Shehata N. (1990) Unexpected products of the reaction of cycloalkylidene(cyano)thioacetamides with arylmethylenemalononitriles: a different novel synthetic route to condensed pyridine-2(1H)-thiones and condensed carbocyclic nitriles. J. Chem. Soc., Perkin Trans. 1, 1, 1267-1270.

27. X-Ray data for $\mathbf{2 g}$ : $\mathrm{C}_{19} \mathrm{H}_{15} \mathrm{ClN}_{4}, M=334.80$, monoclinic system, space group $P 21 / \mathrm{c}, a=13.056$ (3), $b=9.1340(18), c=13.997(3) \AA, \beta=90.11(3)^{\circ} ; V=1669.2(6) \AA^{3}, Z=4$, Dcalcd $=1.332 \mathrm{~g} \mathrm{~cm}^{-3}$, $\mu(\mathrm{Mo}-\mathrm{K} \alpha)=0.236 \mathrm{~mm}^{-1}$, crystal dimension of $0.5 \times 0.25 \times 0.15 \mathrm{~mm}$. The X-ray diffraction measurement was made on a STOE IPDS-II diffractometer with graphite monochromated Mo-K $\alpha$ radiation. The structure was solved by using SHELXS. The Data reduction and structure refinement was carried out with SHELXL using the X-STEP32 crystallographic software package. The non-hydrogen atoms were refined anisotropically by full matrix least-squares on $F^{2}$ values to final $R_{1}=0.0495, w R_{2}=0.1048$ and $S=0.886$ with 225 parameters using 4481 independent reflection $\left(\theta\right.$ range $\left.=2.66-29 \cdot 17^{\circ}\right)$. Hydrogen atoms attached to nitrogen were found in a difference Fourier map and refined isotropically. All other hydrogen atoms were added in idealized positions. The crystallographic information file has been deposited with the Cambridge Crystallographic Data Centre, CCDC 909290.

28. X-STEP32 Version 1.07b, Crystallographic Package; Stoe \& Cie GmbH: Darmstadt, Germany, 2000. 